Revista de Sociología

№ 19 - 2005

Facultad de Ciencias Sociales - Universidad de Chile

(p. 87 - 100)

\title{
EDUCACIÓN, UNIVERSIDAD Y DEMOCRACIA EN CHILE*
}

\section{Carlos Ruiz Schneider ${ }^{* *}$}

Querría desarrollar ante ustedes algunas ideas, de carácter muy tentativo, que surgen de una investigación en curso sobre los proyectos educacionales de la transición democrática en Chiley que significan una cierta continuidad, pero también cambios importantes, en relación al modelo de mercado que impone el régimen militar a partir de la década de 1980.

Para entender cabalmente estos proyectos, me parece importante considerar, en primer lugar, que el modelo democrático de la transición chilena no restaura simplemente la democracia mayoritaria anterior a la crisis de los años 1970, sino que instaura una democracia de nuevo estilo, con muchas limitaciones, que provienen, por una parte, de lo que se ha llamado los "enclaves autoritarios" de la Constitución de 1980 que continúa vigente, y por otra parte, de un estilo de hacer política basado en el modelo de una democracia consensual, que importa también severas restricciones a la lógica igualitaria de las mayorías.

En segundo lugar, hay que mencionar también que, como lo documentan numerosos análisis, a partir del inicio del proceso de transición, se va produciendo una recepción cada vez más positiva del paradigma neo-liberal del mercado por parte de grupos de economistas y dirigentes políticos de la Concertación por la D emocracia, que habían sido, durante la dictadura, sus más importantes críticos. El ex-Ministro Alejandro Foxley expresa estas ideas, o puntos de vista similares, en un ensayo contenido en el libro Políticas económicas y sociales en el C hile democrátion, publicado en 1995: "...el país había vivido un largo período de incertidumbre acerca grado de estabilidad de las reglas del juego en lo político, económico y social. El G obierno del Presidente Aylwin tenía la convicción de que, para impulsar un proceso sostenido de desarrollo, era fundamental reducir esa incertidumbre. Era, por tanto, necesario generar los consensos básicos respecto de las bases del sistema político y económico, y constituir una clase.

\footnotetext{
*Una versión de este ensayo fue publicada anteriormente en el libro: Filosofías de la U niversidad y Conflicto de Racionalidades, compilado por Francisco Naishtat, Ana María G arcía Raggio y Susana Villavicencio, Buenos Aires, Ediciones Colihue, 2001.

${ }^{*}$ Profesor de D epartamento de Filosofía de la Universidad de Chile.
} 
Q uerría desarrollar ante ustedes algunas ideas, de carácter muy tentativo, que surgen de una investigación en curso sobre los proyectos educacionales de la transición democrática en Chiley que significan una cierta continuidad, pero también cambios importantes, en relación al modelo de mercado que impone el régimen militar a partir de la década de 1980.

Para entender cabalmente estos proyectos, me parece importante considerar, en primer lugar, que el modelo democrático de la transición chilena no restaura simplemente la democracia mayoritaria anterior a la crisis de los años 1970, sino que instaura una democracia de nuevo estilo, con muchas limitaciones, que provienen, por una parte, de lo que se ha llamado los "enclaves autoritarios" de la Constitución de 1980 que continúa vigente, y por otra parte, de un estilo de hacer política basado en el modelo de una democracia consensual, que importa también severas restricciones a la lógica igualitaria de las mayorías.

En segundo lugar, hay que mencionar también que, como lo documentan numerosos análisis, a partir del inicio del proceso de transición, se va produciendo una recepción cada vez más positiva del paradigma neo-liberal del mercado por parte de grupos de economistas y dirigentes políticos de la Concertación por la D emocracia, que habían sido, durante la dictadura, sus más importantes críticos. El ex-Ministro Alejandro Foxley expresa estas ideas, o puntos de vista similares, en un ensayo contenido en el libro Políticas económicas y sociales en el Chile democrátion, publicado en 1995: "...el país había vivido un largo período de incertidumbre acerca grado de estabilidad de las reglas del juego en lo político, económico y social. El Gobierno del Presidente Aylwin tenía la convicción de que, para impulsar un proceso sostenido de desarrollo, era fundamental reducir esa incertidumbre. Era, por tanto, necesario generar los consensos básicos respecto de las bases del sistema político y económico, y constituir una clase dirigente que consolidara un sistema democrático y un desarrollo estable" (en Cieplan y Unicef, 1995, p. 110).

En el plano económico, esto se traduce, en opinión de Joaquín Vial, otro de los economistas autores del libro arriba citado, y una autoridad económica importante en el G obierno de Frei, en la necesidad de evitar todo cambio rupturista que pudiera dañar el ahorro y la inversión, lo que genera un compromiso público por mantener el sistema de mercado y la apertura comercial.

Al iniciarse la transición, en un importante discurso en el Encuentro Nacional de la Empresa, en noviembre de 1988, el mismo Alejandro Foxley había sostenido ya que para construir esos consensos en el plano económico-social, era necesario aprender las lecciones de la experiencia, lo que significa en ese momento que "Chile 
tiene que partir de donde está: desde una economía abierta con un proceso de modernización interesante, con un éxito significativo en el plano exportador, con un rol principal para el mercado como mecanismo asignador de los recursos, con un régimen que es básicamente de libertad de precios y con un sector privado que puede desarrollar su labor sin temor a expropiaciones." (Foxley, 1989, p.179).

En palabras de un observador imparcial einteriorizado, como el economista O scar Muñoz, la evolución ideológica de los dirigentes de la Concertación: “...ante la inminencia de un triunfo electoral y ante los signos positivos que mostraba la evolución de la economía, optaron por matizar las críticas del pasado al modelo de mercado, (por) reconocer los aspectos positivos de la estructura económica vigente y, sobre todo, reconocer la importancia de la propiedad privada y de los empresarios en el desarrollo" (Muñoz, 1993, p. 110).

Hay que tomar en cuenta en este análisis además que, a partir de la crisis del petróleo de 1973, y con más fuerza aún en los años 1980, las políticas neo-liberales de ajuste estructural se expanden por todo el mundo, impulsados por los G obiernos Thatcher y Reagan, siendo objeto de recomendaciones especiales por organismos como el Banco Mundial y el Fondo Monetario Internacional a la mayoría de las economías del Tercer Mundo.

¿Cómo perciben el ascenso de esta nueva hegemonía política internacional los dirigentes del nuevo G obierno democrático?. Para José Joaquín Brunner, por ejemplo, la concentración en la lucha contra la dictadura no dejó ver que, además de las transformaciones por el régimen autoritario, "en el resto del mundo se habían producido y estaban ocurriendo cambios que representan, seguramente, la más grande mutación cultural de nuestro siglo...D icho de otra forma, al emerger del autoritarismo, todos los actores tuvimos que considerar con atención los principios constitutivos de ese nuevo paisaje, que pueden sintetizarse con sólo dos términos: democracia y mercados". (Brunner, 1995, p.111). En esto consistiría, para Brunner, el "espíritu de la modernidad" que, sin embargo, sostiene, tampoco es correctamente aprehendido por el discurso neo-liberal.

Uno de los primeros textos que reacciona frente a esta nueva hegemonía mundial, a nivel latinoamericano, es probablemente el documento de CEPAL titulado Transformación productiva con E quidad, de 1990. El modelo económico que subyace a este documento, que se presenta como una alternativa al neo-liberalismo, se sitúa, sin embargo, en opinión de un economista como G onzalo Arroyo, "de una manera realista, al interior de un sistema económico capitalista transnacional y jerarquizado, que permite escasos grados de libertad para las economías nacionales, cada vez 
más interdependientes. Esta dominación capitalista es aún más indiscutible después de la reconversión hacia la economía de mercado, iniciada por los antiguos países socialistas de Europa". ( Arroyo, M ensaje, diciembre de 1990) E sta aceptación realista y relativamente poco crítica de la globalización neo-liberal de la economía mundial incluye, sin embargo, en el documento de CEPAL, algunas reservas que son importantes para entender las políticas educacionales que de ellas derivan. La más importante de esta reservas, tiene que ver con el reemplazo de las simples ventajas comparativas de América Latina en el mercado mundial y que se reducen, a menudo, en el esquema neo-liberal anterior a los bajos salarios, por las llamadas ventajas competitivas, ligadas a aumentos en la productividad del trabajo. En este sentido, el documento de CEPAL vuelve a insistir en la temática del capital humano, que había sido central para las teorías del desarrollo en los años 1960, centradas en la industrialización sustitutiva, y que había estado ausente del discurso y las políticas neo-liberales del régimen militar.

Las políticas educativas que derivan de los programas de ajuste estructural a nivel mundial y que se recomiendan a los países del Tercer Mundo, no son en realidad muy diferentes de las políticas neo-liberales que se habían aplicado en Chile bajo el régimen militar. Estas últimas aparecen, por lo demás, constantemente citadas, a título de ejemplos paradigmáticos de políticas del futuro, por organismos como el Banco Mundial. Es, entonces, este tipo de visión el que conforma el marco conceptual y la filosofía de base de los más importantes organismos de financiamiento internacional de proyectos educativos por lo que no es extraño que se genere una fuerte presión para que los modelos de la privatización y el mercado sigan estando presentes en las políticas educativas chilenas de los 1990.

Uno de los puntos centrales de estas políticas de alcance mundial, que sirven de fundamento a recomendaciones, también a escala mundial, es que la educación no puede estar hoy completamente a cargo del Estado. En la interpretación de una analista como Teresa Mariano Longo, lo que se busca es "evitar la inmovilización de recursos importantes que podrían encontrar una utilización más rentable en el mercado" (Mariano Longo, "Politiques d'ajustement structurel et professionalité des enseignants", Paris, UNE SCO, 1993, p. 7). En esta perspectiva, y reconociendo la importancia de la educación para el crecimiento económico, en la línea de los trabajos sobre "capital humano", se constata que la necesaria reducción del aporte estatal, si quiere ser efectiva, tiene que significar también probablemente un corte en los salarios de los maestros, ya que estos representan el más alto porcentaje del gasto educacional. En este sentido, los informes del Banco Mundial, por ejemplo, recomiendan acciones como la exclusión de los profesores de la función pública, acercar al mínimo la base fija de los salarios y desarrollar una política de primas por 
desempeño, descentralizar los servicios educacionales para hacer más difícil la acción de los sindicatos nacionales, bajar el costo, de la formación de los maestros y, por último, el aumento del número de alumnos por clasey del tiempo de trabajo de los profesores.

En el caso de la Universidad, las perspectivas que derivan de estos organismos tienen características específicas. En un informe del Banco Mundial titulado L a E nseñanza Superior. L as lecciones derivadas de la ex perienda y publicado en 1995, leemos, por ejemplo, que la función de las Universidades es educar a los futuros dirigentes y preparar las capacidades técnicas de alto nivel que constituyen la base del crecimiento económico.(Banco Mundial, 1995, p. VII).

La Enseñanza Superior, se nos dice en el documento, "reviste capital importancia para el desarrollo económico y social. Las instituciones de nivel terciario tienen la responsabilidad principal de entregar a las personas los conocimientos que se requieren para desempeñar cargos de responsabilidad en los sectores público y privado. Estas instituciones entregan nuevos conocimientos a través de la investigación, sirven de medio para transferir, adaptar y divulgar los conocimientos que se generan en otras partes y apoyan a los gobiernos y a los círculos empresariales prestándoles servicios de asesoría y consultoría. En la mayoría de los países, las instituciones de educación postsecundaria desempeñan también importantes funciones sociales puesto que contribuyen a plasmar la identidad nacional y sirven de foro para un debate pluralista”(Ibíd., p. 1).

Como se ve en estos pasajes, el tema central, a través del cual el Banco conceptualiza a la Educación Universitaria es el de su contribución al crecimiento económico. Unas páginas más adelante, el mismo texto precisa que "la contribución de la enseñanza superior al desarrollo de los países es muy amplia. Las inversiones en este subsector pueden influir de manera importante en el crecimiento económico de un país. Las instituciones de nivel terciario tienen la función principal de capacitar al personal profesional, entre ellos, los gerentes, científicos, ingenieros y técnicos que participan en el desarrollo, la adaptación y la difusión de las innovaciones en la economía nacional” (Ibíd., p. 17).

A pesar de la importancia concedida a la educación superior para el crecimiento económico, el documento del Banco asigna una baja prioridad a la inversión pública en el sector. Sostiene que "en el sector de educación hay pruebas de que las inversiones en el sector terciario tienen tasas de rentabilidad social más bajas que las inversiones en enseñanza primaria y secundaria, y que las inversiones en educación básica pueden también incidir más directamente en la reducción de la pobreza, puesto que tienden a mejorar la igualdad de los ingresos”. (Ibíd., p. 14). 
En función de estas consideraciones, según el documento, la función de los préstamos del Banco en este campo no podría ser sino contribuir al desarrollo de reformas de política que apunten a que el sector funcione con un menor costo público. En los términos del libro": Se seguirá dando prioridad a los países dispuestos a adoptar un marco de políticas en materia de educación superior que haga hincapié en la estructura diferenciada de las instituciones y una base de recursos diversificada y mayor importancia a los proveedores y al financiamiento privados."(Ibíd., p. 14).

En síntesis en este punto, la recomendación del Banco es, que en los países en desarrollo, en particular en los que tienen todavía problemas en el acceso, calidad y equidad a la educación básica y media, la educación superior no debiera tener la preferencia en la utilización de recursos fiscales adicionales para el sector educación.

La experiencia de la educación superior en Chile muestra precisamente, para el Banco, que el sector puede funcionar bien, ser diversificado y experimentar crecimiento, incluso cuando el gasto público por estudiante disminuye. Hay que recordar aquí, sin embargo que las evaluaciones que ha hecho recientemente el mismo Banco en 1998 de la Educación Superior chilena distan de ser halagadoras. En todo caso, lo que parece ser el caso, es que es el modelo global lo que cuenta, más allá de sus imperfecciones puntuales.

¿Cuáles son, entonces, en síntesis, las propuestas de una organización como el Banco Mundial para la educación superior?

En primer lugar habría que señalar que las recomendaciones del Banco descartan la Universidad humboldtiana, basada en la integración entre docencia e investigación: "El modelo tradicional de Universidad europea de investigación, con sus programas en un sólo nivel, ha demostrado ser costoso y poco apropiado para satisfacer las múltiples demandas del desarrollo económico y social, al igual que las necesidades de aprendizaje de un estudiantado más diverso. La introducción de una mayor diferenciación en la enseñanza superior, es decir, la creación de entidades no universitarias y el aumento de instituciones privadas puede contribuir a satisfacer la demanda cada vez mayor de educación postsecundaria y hacer que los sistemas de enseñanza se adecuen mejor a las necesidades del mercado de trabajo." (Ibíd., p.31).

A parte fomentar una mayor diferenciación y el desarrollo de instituciones privadas, las recomendaciones del Banco apuntan también a la diversificación de las fuentes de financiamiento, (lo que quiere decir, sobre todo, que los estudiantes deben pagar por sus estudios), a una estrecha vinculación entre el financiamiento fiscal y los resultados, y también a la construcción de un sistema de educación 
superior equitativo, sin que sea muy claro cómo pueda conseguirse mayor equidad en un sistema para cuyo desarrollo se confía cada vez más en el mercado. Pero, por sobre todo estas recomendaciones apuntan a una redefinición completa del rol del Estado en la enseñanza superior.

Hay dos razones fundamentales que justifican, para el Banco, que, de todos modos, haya por lo menos alguna intervención del Estado en el financiamiento de la educación superior. La primera razón tiene que ver con el hecho de que el financiamiento de la investigación básica y de la transferencia tecnológica por la empresa privada no sería óptimo. La segunda razón tiene que ver con carencias relacionadas con la equidad: la imperfección de los mercados de capital, limita la capacidad para obtener préstamos adecuados para educación, lo que perjudica particularmente a las personas talentosas pertenecientes a los grupos más desfavorecidos económicamente.

En estas condiciones, el Estado debe intervenir en el área, pero su intervención, en los países en desarrollo, sobrepasa ya con creces lo que debe ser.

Según el Banco, esta intervención debiera centrarse no en una función de control directo, sino en la elaboración de un marco coherente de políticas para el área, en desarrollar formas de apoyo consistentes en incentivos e instrumentos orientados al mercado para la aplicación de las políticas, y en hacer posible una mayor autonomía administrativa de las instituciones públicas.

El diagnóstico que hace el Banco es que, a menos que se hagan reformas para mejorar los resultados de la enseñanza superior, “...muchos países están destinados a entrar al siglo XX I con una preparación insuficiente para competir en la economía mundial, donde el crecimiento dependerá cada vez más de los conocimientos técnicos y científicos". (Ibíd., p. 28).

En esta perspectiva, hay para el Banco sobre todo dos enemigos fundamentales de estas reformas: el activismo de los estudiantes y la ineficiencia de los gobiernos. En este sentido, sostiene el documento: "La experiencia demuestra que...no deben subestimarse las dificultades políticas.. En los países que tienen sistemas débiles de go bierno, los estudiantes con reivindicaciones - y las habrá si se reducen los subsidios y los privilegios - pueden representar una amenaza a la estabilidad política. (Ibíd., p. 29).

En el caso chileno, a partir de los años 1990 y con posterioridad a la instalación de un gobierno democrático, haido perfilándose un modelo educacional que mantiene una importante continuidad con el modelo neo-liberal del mercado, 
que, por otra parte, no ha sido posible erradicar completamente, pero que incluye también algunos cambios importantes. Este modelo se expresa, por ejemplo, en el Informe L os desafíos de la educación chilena frente al siglo X X I de la Comisión Nacional para la Modernización de la Educación. Algunas de estas ideas ya se encontraban presentes en el Informe de la Comisión de Estudio de la Educación Superior titulado U na política para el desarrollo de la E ducación Superior en la década de los $\mathrm{N}$ oventa. Ambos textos se relacionan, a su vez, con documentos de CEPAL como Transformación productiva con E quidad de 1990 y sobre todo con el texto sobre $E$ ducación y conocimiento: eje de la transformación productiva con equidad, editado por CEPAL y UNESCO en 1992.

Como lo señalábamos más arriba, estos cambios significan un nuevo protagonismo para el campo educativo, ya que la globalización de la economía de mercado impone como condición de sustentabilidad de modelos económicos que se sabe que son inequitativos y que van a continuar siéndolo, el aumento de la productividad y la capacitación de las fuerzas productivas a través de la educación, la que resultará ser así la única fuente de disminución de la pobreza y las crecientes desigualdades sociales, ya que las políticas redistributivas estatales están completamente excluidas por este estilo de política económica global.

Este es el interés que revisten, para estas visiones, los países del Este asiático. Lo que los expertos gubernamentales, sobre todo los economistas, subrayan en estos modelos, es que ellos parecen demostrar que su crecimiento económico espectacular está ligado a un aumento sin precedentes del gasto en educación. En términos del trabajo de CEPAL y UNESCO sobre E ducación y onocimiento: E je de la transformación productiva on equidad, se sostiene, por ejemplo, que la incorporación sistemática del progreso técnico constituye el pivote fundamental de la transformación productiva y que las ventajas competitivas de los países deben descansar sobre el aumento de la productividad, que es un efecto de la incorporación del progreso técnico. Entre los factores que influyen en la difusión del progreso técnico están el fortalecimiento de la base empresarial, la infraestructura tecnológica, la apertura de la economía y, en especial, la formación de recursos humanos y los incentivos y mecanismos que favorecen el acceso y la generación de nuevos conocimientos. Más precisamente, la idea formulada por Peter $D$ rucker de que nos encaminamos a una forma de sociedad cuyo centro es el conocimiento, está en la base de muchos de estos diagnósticos. (Cox y Courard, 1996). Al convertirse así el conocimiento en el elemento central del nuevo paradigma productivo, la educación y la transformación educativa pasan a ser factores fundamentales para alcanzar altos niveles de competitividad y crecimiento económico. Esto representa un elemento de cambio frente al modelo de mercado puro y duro del régimen militar 
y también, hasta cierto punto, trae consigo modificaciones al modelo del Banco Mundial.

En lo que se refiere a la Universidad, las bases de estas nuevas concepciones están bastante bien delineadas en documentos como el informe de la Comisión de Estudio de la Educación Superior titulado U na política para el desarrollo dela E ducación Superior en la década de los $\mathrm{N}$ oventa, que mencionábamos más arriba.

En el estilo de innumerables otros informes de "expertos", un estilo que ha caracterizado el estilo elitista de hacer política de los G obiernos de la transición Chilena, el documento que comentamos está elaborado a partir de una invocación al consenso, que reemplaza su discusión democrática. Para José Joaquín Brunner, Presidente de la Comisión que elaboró el estudio, “...los documentos preparados Reflejan un amplio y sólido acuerdo intelectual, técnico y de políticas para el sector." (Informe,....1990, p. VIII). En opinión de Ricardo Lagos, a la sazón Ministro de Educación, se está tratando de elaborar "...una política y una legislación para la Educación Superior que represente un genuino acuerdo para todos los chilenos" ( Ibid.,p.X I). A esto agrega el Ministro que se tiene la convicción de que "...las materias educacionales interesan a todo el país y comprometen el futuro de todos nosotros. Por ello que las políticas en este ámbito deben ser políticas concordadas". (Ibid, p. IX).

En realidad, este estilo consensual y elitista, junto a un llamado a una especie de criterio de logro y a un principio de realidad, frente a las utopías del pasado, constituyen el tono y el contenido central del discurso del Informe. Según José Joaquín Brunner, en todos los países y no sólo en Chile, "está llegando a su fin el ciclo...en que podía disponerse de recursos financieros con relativa abundancia y sin vincular su asignación a programas, a metas evaluables y a criterios de desempeño y rendimiento". (Ibid, p. VIII). En este mismo sentido, y en un texto relacionado con el del Informe, Brunner sostiene que la crisis actual de la Educación Superior en América Latina, "hace imperativo abandonar los viejos modelos benevolentes e interventores" aunque también el más reciente modelo de "desregulación orientado hacia un mercado de la educación superior". (Brunner, 1990, p. 70).

Para el mismo autor, todavía predomina en América Latina un temario de discusión en torno a la enseñanza superior que es " heredero de los 60, así como sus formulaciones corresponden a una realidad ya superada en la práctica...Las antiguas retóricas, por ejemplo, en torno a la educación superior libre y gratuita para todos, a las formas electorales de participación en el gobierno de las universidades, a la universidad comprometida con el cambio social (pero aislada de 
su medio y de escasa calidad y rendimiento), como tantas otras retóricas que se han quedado atrapadas en el pasado, no iluminan ya los problemas del presente ni tienen capacidad de crear el futuro. E sas retóricas son parte de un "progresismo" que se quedó encerrado mental e ideológicamente en sus propias producciones, sin capacidad de abrirse a las nuevas circunstancias, a los desafíos contemporáneos y a las exigencias del tiempo que viene." (Ibíd., p. 76).

El nuevo modelo de educación superior que se propone para el país, en palabras del Ministro de Educación, debe apuntar al hecho que: "En último término la inserción que queremos para Chile en un mundo internacional cada vez más competitivo, donde la eficiencia es el único mecanismo para poder insertarse plenamente en él, está determinada por la capacidad del sistema educacional que seamos capaces de crear... No existe otra lección que podamos aprender de aquellos países que hoy consideramos exitosos, si no es que ellos salieron adelante por su capacidad de exportar valor agregado. En último término esto significa exportar conocimiento. Y detrás del conocimiento está una política educacional amplia y comprensiva." (Informe, p. XII).

La política propuesta por el Informe apunta a 6 objetivos principales: 1) Consolidar la base institucional de la educación superior; 2) Impulsar un desarrollo cualitativo de la matrícula;3)A segurar la calidad y equidad del sistema; 4) Fomentar la creación científica y la creación cultural; 5) Incrementar y diversificar el financiamiento y 6) Perfeccionar el marco legislativo. Entre estos puntos, los más relevantes se refieren al pago de los estudios, al rol del mercado y al rol del Estado en el sector.

En relación al tema del pago de los aranceles por los estudiantes, el texto del Informe es claro y taxativo: "Los estudiantes deben concurrir a financiar en la medida de sus posibilidades económicas el costo de la enseñanza que reciben la cual les permitirá más adelante obtener ingresos provenientes del ejercicio de una profesión o actividad certificada por el respectivo título técnico. Si no se procediera de esta forma se violaría el principio de equidad, sobre todo si se considera que en Chile sólo 18 de cada 100 jóvenes del respectivo grupo de edad ingresan a algún establecimiento de educación superior". (Ibíd., p. 59).

El rol del mercado en la educación superior es analizado por el documento en términos bastante cautos, pero sustancialmente positivos. La existencia de universidades privadas, orientadas al mercado, que son una creación del régimen militar, no es cuestionada en ningún momento, y a la inversa, se intima a las universidades públicas a aumentar la cuota de sus recursos que provienen de la 
empresa privada. Así por ejemplo, el Informe propone que durante los próximos años, el Estado realice un incremento de su apoyo a la educación superior, hasta llegar a un monto equivalente al 1\% del PGB, pero éste aumento, sostiene el documento, "deberá ir acompañado por el esfuerzo de los propios establecimientos para mantener e incrementar los recursos que obtienen por la vía de venta de servicios y de contratos de investigación, así como una política más activa para captar recursos adicionales del sector privado aprovechando al efecto el esquema de exenciones tributarias". (Ibíd., p. 65). Para los autores del documento, debe asegurarse que "una proporción significativa de las asignaciones fiscales a la educación superior sea canalizada mediante mecanismos que incentiven a las instituciones receptoras a incrementar la calidad, la eficiencia y la relevancia de las actividades que acometen. La competencia fundada en valores académicos y ejercida a través de proyectos intelectuales puede ser, en el contexto de las políticas aquí propuestas, un principio positivo para la vida de las instituciones de educación superior. Pretender, en cambio, que la "competencia de mercado" pudiera convertirse en el único criterio por el cual ellas deberían regirse conduciría a la larga a efectos perversos..." (Ibíd., p. 61-62).

Como puede vislumbrarse en los pasajes anteriores, el documento asigna de todos modos un rol importante aunque disminuido e indirecto, al Estado en la educación superior. El Informe propone, entre otras materias, la creación de un Consejo de Educación Superior, cuyo objetivo es controlar y garantizar la seriedad de los estudios ofrecidos por el sistema. El Estado, asegura el documento, "cumple un papel insustituible en el financiamiento de la educación superior. A él le corresponde asegurar que exista en el país una adecuada distribución de oportunidades de nivel superior, en la cantidad y con la calidad que requiere el desarrollo nacional, y que se mantengan y amplíen las capacidades de investigación científica y tecnológica y de creación en todas las áreas del arte, las letras y las humanidades... El Estado debe contribuir al financiamiento directo de aquellas instituciones que forman el núcleo más sólido de la educación superior del país de modo de garantizar su estabilidad institucional, la presencia en el país de un grupo permanente de académicos del más alto nivel y el desarrollo de una variada gama de actividades de investigación, de formación avanzada y de extensión, a lo largo del territorio nacional."(Ibíd., p. 66). Sin embargo, el financiamiento público a la educación superior se presenta en el documento como cada vez más condicionado por criterios de rendimiento y eficiencia y, sobre todo, como complementando los recursos que las entidades públicas puedan conseguir en la empresa privada.

En síntesis, el nuevo modelo de educación superior que surge de estos documentos puede ser descrito, como lo hace por ejemplo José Joaquín Brunner, en términos de un sistema dotado de "una regulación liviana y eficaz". La regulación 
del sistema "desde la distancia" "implica necesariamente, aceptar que el Sistema de Educación Superior opere con autonomía y en buena medida de acuerdo a exigencias de mercado, sea del mercado de demandas estudiantiles, del mercado de oferta de puestos, del mercado laboral-académico, de los mercados para la captación de recursos y del mercado de reputaciones institucionales...Pero significa reconocer además, y al mismo tiempo, que la coordinación proporcionada por los mercados no puede hacerse cargo de la evolución y el desarrollo del sistema, debido a las "cegueras" que produce, a las distorsiones que introduce y a las inquietudes que genera. De allí que deba definirse, asimismo, un rol amplio y productivo para el Estado en su relación con la enseñanza superior..." (Brunner, 1990, p. 76).

En términos del mismo autor, la educación superior debería pensarse en función de una nueva relación con el Estado, al que denomina "Estado evaluativo", esto es, "un Estado que parte por reconocer la autonomía y la diversidad de las instituciones de la enseñanza superior y el carácter de bien público de los servicios que ellas ofrecen, y que por tanto contribuye a su financiamiento, pero que a la vez se hace cargo -por medios diversos- de promover la evaluación y auto evaluación de las acciones y los resultados obtenidos por las universidades y de orientarlas "desde la distancia", velando por los intereses de toda la sociedad".(Brunner, 1990, p.70).

Para el mismo autor, esto se expresa en una política que supone al menos los siguientes elementos claves:1) Una evaluación continua de las instituciones y actividades, con participación de pares, expertos del sector público y "representantes del sector privado empresarial"; 2) La diversificación del financiamiento de las instituciones y actividades, "buscando trasladar el centro de gravedad hacia modalidades de financiamiento público y privado sensibles a la demanda y basadas, en el caso de las asignaciones fiscales, en contratos de desempeño y/ o de metas, en resultados evaluados, en concursos de proyectos, en licitaciones competitivas, 0 en "fórmulas" que incentiven la consecución de objetivos de interés público; y 3) Una conducción estratégica de los sistemas de educación superior y de "I\&D" (sic) "en función del desarrollo de capacidades nacionales que permitan aumentar la integración social y la competitividad de nuestras redes de producción, comercio y conocimiento."(Brunner, 1990, p.190).

Con razón observa Brunner que resulta polémico sostener que en Chile se ha avanzado en algunas de esas direcciones, lo que ha llevado a instituciones como el Banco Mundial, a presentar las transformaciones de la educación chilena como "un modelo 0 experiencia que vale la pena estudiar e imitar". (Ibíd..., p. 190). Con algunas reservas, le parece sin embargo al autor que "nos movemos, básicamente 
en la dirección correcta” y que "el sistema se ha vuelto globalmente más eficiente y dinámico, pero en él tienden a pesar ahora, quizá desmedidamente, los intereses de corto plazo del mercado de demandas estudiantiles." (Ibíd., p. 191).

Como creo que permiten apreciarlo los pasajes que hemos comentado, hay importantes elementos de cambio, pero también una sustancial continuidad entre estas nuevas propuestas educacionales y el modelo de mercado en educación que, basado en unos pocos artículos de Milton Friedman, los militares impusieron en Chile en la década del 80. Esta continuidad, especialmente visible en el caso de la educación superior, no es sólo el resultado de una imposición por la fuerza, sino que incorpora también importantes elementos de una crítica anti-utópica que apunta a los modelos educacionales anteriores, incluyendo las teorías del desarrollo en educación.

Como en el caso de estas teorías del desarrollo, basadas en las ideas sobre el "capital humano", volvemos a ver aquí también una dependencia predominante de la educación respecto de las exigencias de la producción y la economía globalizada. En el Informe G ubernamental L os desafíos de la E ducación chilena frente al siglo X X I, esta funcionalidad de la educación respecto de la esfera productiva es mayor aún, ya que en él se sostiene que la educación, a través del incremento del capital humano de los trabajadores y de la productividad, debe constituir la base fundamental de toda política de mejoramiento salarial. La educación aparece, en muchos documentos oficiales, investida incluso de una nueva función, la de ser el único medio eficaz para hacer frente a la pobreza, en el marco de una economía capitalista liberal globalizada que excluye la intervención redistributiva de parte del Estado. D e hecho, los mayores esfuerzos reales en favor de la equidad, en las políticas públicas de los gobiernos de la transición, es el que se lleva a cabo en torno a las políticas educativas como el MECE o el Programa de las 900 Escuelas.

A hora bien, en general, de esta manera tiende a acrecentarse la funcionalidad de la educación respecto del sistema económico, porque es indudable que el tipo de educación que puede ligarse directamente con aumentos de productividad es la que se relaciona con la capacitación productiva. Por otra parte, es también probablemente este tipo de educación el que va a encontrar más financiamiento privado.

Pero también así nos alejamos de una solución realista del problema de la pobreza y la desigualdad, ya que esta tiende, a lo sumo, a ser reproducida a otro nivel por los sistemas educacionales, si no hay esfuerzos redistributivos reales y paralelos en toda una serie de otras esferas, como el trabajo y la salud, por lo menos. 
100 Revista De Sociología

№ $19-2005$

Aquí parece que nos encontramos, sobre todo, con los efectos de la transnacionalización y la globalización neo-liberal de las economías, de las que deriva como un axioma que todo intento redistributivo, toda reforma tributaria etc. está excluida de la agenda pública por principio, en función de la mantención de reglas del juego atractivas para el capital globalizado. Si esto es así, por considerables que sean los esfuerzos hechos en favor de la equidad por los proyectos educativos, ellos van a ser siempre insuficientes, si constituyen, como probablemente constituyen hoy, prácticamente las únicas políticas públicas orientadas a revertir la pobreza y las crecientes desigualdades que caracterizan hoy a la sociedad chilena.

\section{Referenciasbibliográficas}

Banco Mundial (1995) L a enseñanza superior. L as lecciones derivadas de la ex periencia.

Brunner, José Joaquín (1990) “ Universidad, sociedad y Estado en los 90” en N ueva Sociedad, No 107, mayo-junio.(1995)

"Los G obiernos de la Concertación: social-progresismo versus neo-liberalismo" E studios CIE PL A N , No 41. (1997)

"La educación superior chilena como objeto de análisis y de políticas" en E studios Sociales, No 91. CEPAL (1990) Transformación productiva con equidad.

CEPAL-UNESCO (1992) E ducación y œnocimiento: eje de la transformación productiva œn equidad. CIEPLAN y UNICEF (1995) Políticas económicas y sociales en el Chile democrátion.

Comisión de Estudio de la Educación Superior (1990) U na política para el desarrollo de la E ducación Superior en la década de los noventa.

Comité Técnico Asesor del Diálogo Nacional sobre la Modernización de la Educación Chilena (1994) L os desafíos de la E ducación C hilena frente al siglo X X I.

C.Cox y H.Courard, (1996) “El Estado, el mercado y la educación en los 90" en ENERSIS, E ducación en Chile: un desafío de calidad.

Foxley, Alejandro (1989) "Bases para el desarrollo de la economía chilena: una visión alternativa" en E studios C IE PL A N , N 026.

Mariano Longo, Teresa (1993) Politiques d'ajustement structurel et professionnalitédes enseignants, UNESCO, Paris.

Muñoz, O scar y Carmen Celedón (1993) "Chile en transición: estrategia económica y política “. E studios CIE PL A N, N 037. 